\title{
Aves y mamíferos de bosque altoandino-páramo en el páramo de Rabanal (Boyacá-Colombia)
}

\author{
Birds and Mammals of High Mountain Ecosystems in the Rabanal Paramo \\ (Boyacá-Colombia)
}

W. Medina ${ }^{\mathrm{a}, *}$

D. C. Macana García ${ }^{b}$

F. Sánchez ${ }^{\mathrm{c}}$

Recepción: 05-ene-15

Aceptación: 15 -jul-15

\begin{abstract}
Resumen
El páramo de Rabanal en Colombia es considerado un reservorio hídrico para la región del altiplano cundiboyacense, y ha sufrido un grave deterioro por actividades humanas. Los inventarios de la biodiversidad son útiles para ejecutar planes adecuados de conservación de especies y sus ecosistemas; por ello, se realizó un inventario de aves y mamíferos del sector Matanegra en Rabanal, municipio de Ventaquemada (Boyacá). Para el registro de aves se emplearon recorridos y puntos de observación, detecciones ad libitum y redes de niebla. Para el estudio de mamíferos se usaron recorridos de observación, trampas de captura en vivo y búsqueda de rastros. Se registraron 57 especies de aves y 14 de mamíferos, entre las que se destacan taxones con distribución restringida (Anas andium, Colinus cristatus bogotensis, Fulica americana columbiana, Gallinago nobilis, Eriocnemis cupreoventris, Conirostrum rufum, Atlapetes pallidinucha, Myioborus ornatus, Sporagra spinescens, Cryptotis thomasi y Thomasomys niveipes); en categoría de amenaza (Cuniculus taczanowskii y Nasuella olivacea), y se presentan 39 nuevos registros para la zona. El número de especies en Rabanal es bajo, comparado con áreas protegidas en Boyacá y Cundinamarca; sin embargo, a pesar de la intervención antrópica, Rabanal aún contiene elementos típicos de ecosistemas andinos. Se recomienda adelantar procesos pedagógicos con la comunidad local para mitigar los impactos sobre el páramo.
\end{abstract}

Palabras clave: Andes, altiplano cundiboyacense, avifauna, inventarios de biodiversidad, mastofauna.

\footnotetext{
Abstract

The Rabanal Paramo is an important water source for the departments of Cundinamarca and Boyacá, but its natural ecosystems have been profoundly modified by human activities. Biodiversity inventories are useful tools to propose conservation and management plans for the species and the habitats they rely on. Therefore,

${ }^{a}$ Escuela de Biología, Facultad de Ciencias Básicas, Universidad Pedagógica y Tecnológica de Colombia.

*Autor de correspondencia: wilderson.medina@uptc.edu.co

${ }^{\mathrm{b}}$ Asociación Ornitológica de Boyacá-Ixobrychus.

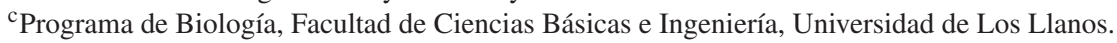


an inventory of birds and mammals of Matanegra zone, Rabanal Paramo, municipality of Ventaquemada (Boyacá) was conducted. Point counts, transects, detections ad libitum, and mist nets were used to record birds; and transects, track searches and live traps were used for mammals. 57 species of birds and 14 of mammals were recorded. Several species have restricted distributions (Anas andium, Colinus cristatus bogotensis, Fulica americana columbiana, Gallinago nobilis, Eriocnemis cupreoventris, Conirostrum rufum, Atlapetes pallidinucha, Myioborus ornatus, Sporagra spinescens, Cryptotis thomasi y Thomasomys niveipes), are endangered (Cuniculus taczanowskii y Nasuella olivacea), or have not been previously reported in the area (39 species). The number of species for Rabanal is low compared with protected neighboring areas in Boyacá and Cundinamarca. However, despite human-caused perturbations, Rabanal contains typical elements of high mountain ecosystems. Conservation measures such as the development of environmental education projects with local communities to mitigate the impacts on the paramo are recommended.

Key words: Andes, Birds, Biodiversity inventories, Boyacá-Cundinamarca high plateau, Mammals.

\section{Introducción}

Los ecosistemas de alta montaña colombianos son áreas prioritarias para la conservación, debido a la riqueza biológica que albergan, a su alto grado de endemismo y a los bienes y servicios que nos brindan [1-3]. Esta prioridad se hace mayor dadas las amenazas por las actividades humanas y el cambio climático global, las cuales persisten en lo alto de Los Andes [4]. También, estos ecosistemas están entre los menos estudiados en el Neotrópico, lo que dificulta su manejo y conservación [5]. Un ejemplo de lo anterior en las montañas colombianas se observa en el páramo de Rabanal, una reserva hídrica para el altiplano Cundiboyacense, el cual se halla sometido a minería, plantación de especies exóticas, actividades agrícolas, creación de represas, pastoreo y quemas [6-8]; adicionalmente, allí la caza parece ser uno de los principales agentes de pérdida de fauna local, afectando principalmente a aves y mamíferos [9]. Por lo tanto, existe una reconocida necesidad de evaluar la diversidad biológica de estas áreas, para así establecer adecuados planes de manejo y conservación de especies [10, 11].

En el altiplano Cundiboyacense se han realizado estudios sobre sus aves [10-15] y mamíferos [16, 17, 18]; sin embargo, continúa siendo escaso el conocimiento científico de estos grupos para la región, y en particular para el páramo de Rabanal, donde, con base en entrevistas con la comunidad y observaciones de campo, se ha indicado la probable ocurrencia de algunas especies y que otras están posiblemente extintas $[9,19,20]$. Por lo tanto, es necesario conocer el estado actual de la diversidad de aves y mamíferos en esta área, dada la importancia hídrica que representa para la región y el país, y por el papel que los integrantes de estos grupos juegan en la conservación de la integridad funcional de los ecosistemas de alta montaña. Con base en lo anterior, el objetivo de este estudio fue realizar una caracterización de aves y mamíferos en el sector Matanegra del Páramo de Rabanal, en el municipio de Ventaquemada. En este trabajo, adicionalmente, se brindan anotaciones sobre el comportamiento de algunas especies, y se sugieren alternativas para el desarrollo de planes de conservación de las especies y sus hábitats.

\section{Materiales y métodos}

\section{1 Área de estudio}

El estudio se desarrolló en el sector Matanegra del páramo de Rabanal, en el municipio de Ventaquemada, departamento de Boyacá (coordenadas $5^{\circ} 24^{\prime} \mathrm{N}$ $73^{\circ} 32^{\prime} \mathrm{W}$ ), entre los 3030 y los $3350 \mathrm{~m}$ de altitud (figura 1). Allí se encuentran fragmentos de bosque altoandino (6-7 ha) de 10-12 $\mathrm{m}$ de altura en el estrato arbóreo, afectados por entresaca y ubicados en terrenos con pendientes de $10-30^{\circ}$. Dichos fragmentos están dominados por especies de las familias Melastomataceae, Lauraceae, Rubiaceae, Rosaceae, Ericaceae y Poaceae, y colindan con áreas de páramo azonal que presentan un alto nivel freático. Las áreas de páramo azonal (frailejonal) están dominadas por Espeletia murilloi y E. boyacensis, y por herbáceas como Puya goudotiana, P. trianae, Paepalanthus sp., Calamagrostis effusa, Valeriana pilosa, Arcytophyllum muticum y Eryngium humboldtii, y por el musgo Sphagnum sp. Adyacentes a los fragmentos de bosque y al páramo azonal persisten áreas de pajonal (Calamagrostis effusa), en las cuales se encuentran inmersos el frailejón endémico, Espeletiopsis rabanalensis y E. argentea, y arbus- 
tos de las familias Ericaceae y Asteraceae. Hay dos humedales naturales, Laguna Verde y Laguna Cristal, con una extensión de 3,69 ha y 0,61 ha, respectivamente [20].

Los sistemas que son resultado de actividades humanas comprenden áreas de cultivo de papa (Solanum spp.) y zanahoria (Daucus carota), potreros para ganadería, plantaciones de especies exóticas (Pinus patula y Acacia melanoxilon) y plantaciones de aliso (Alnus acuminata); estas últimas establecidas en áreas que anteriormente estaban sometidas al cultivo de papa. Según pobladores locales, todas las áreas mencionadas están conectadas por caminos de herradura.
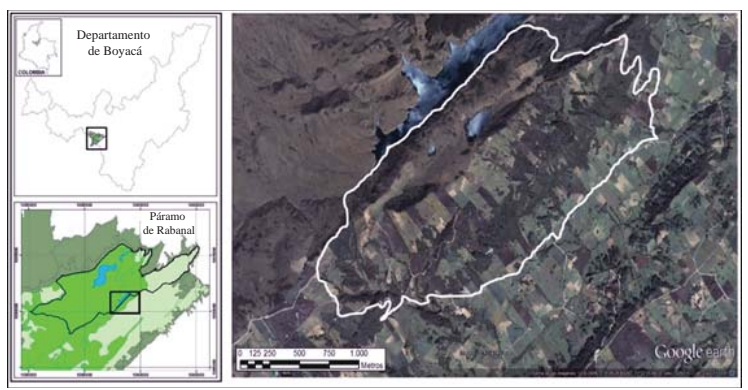

Figura 1. Sector Matanegra (perímetro en blanco) en el páramo de Rabanal (Boyacá-Colombia). La zona es un mosaico de ecosistemas nativos y transformados por las actividades humanas. Imagen modificada de Google Earth, 2009.

\subsection{Métodos}

Se estudiaron las aves y los mamíferos durante tres épocas: abril, agosto-septiembre de 2013 y eneromarzo de 2014. Los muestreos fueron hechos principalmente en fragmentos de bosque nativo y en áreas adyacentes del páramo azonal. Las aves fueron detectadas visual y auditivamente, en recorridos de observación y puntos de conteo [21,22] realizados entre las 05:30 h y las 17:00 h, dentro de las áreas mencionadas. Para acceder a los sitios se recorrieron principalmente caminos de herradura; también se visitaron áreas próximas que incluían humedales, pajonales, matorrales, áreas reforestadas con aliso o con especies exóticas, cultivos, pastizales y cerca de viviendas habitadas o abandonadas. Los recorridos por estas áreas fueron aprovechados para hacer observaciones adicionales y conocer la fauna asociada; así, en estas áreas el esfuerzo de muestreo fue menor (tabla 1). Las detecciones auditivas se hicieron mediante grabación de vocalizaciones para apoyo en la identificación. Adicionalmente, se abrieron tres redes de niebla (12,5 m; ojo de malla de $30 \mathrm{~mm}$ ) durante las 06:00 h y las 16:00 h, para un esfuerzo de 77,5 horas por red durante abril y agosto de 2013, únicamente en la transición entre bosque secundario y frailejonal, y en áreas reforestadas con vegetación exótica. También se registraron aves en cámaras trampa dispuestas para la detección de mamíferos.

Tabla 1. Métodos y esfuerzo de muestreo de aves y mamíferos en distintas coberturas de vegetación, en el sector Matanegra, vereda Montoya del municipio de Ventaquemada (Boyacá, Colombia).

\begin{tabular}{|c|c|c|c|c|c|c|}
\hline Grupo & Método & & $\begin{array}{l}\text { be } \\
\mathrm{F}\end{array}$ & $\begin{array}{l}\text { tura } \\
\text { VE }\end{array}$ & (b) & $\begin{array}{l}\text { Esfuerzo } \\
\text { de muestreo }\end{array}$ \\
\hline \multirow[t]{2}{*}{ Aves } & Puntos de conteo & $\mathrm{x}$ & $\mathrm{x}$ & & & $13 \mathrm{~h}$ en $7,8 \mathrm{~km}^{2}$ \\
\hline & Redes de niebla & $\mathrm{x}$ & $\mathrm{x}$ & $\mathrm{x}$ & & $77,5 \mathrm{~h} / \mathrm{red}$ \\
\hline \multirow[t]{3}{*}{ Mamíferos } & $\begin{array}{l}\text { Trampas } \\
\text { Sherman }\end{array}$ & $\mathrm{x}$ & $\mathrm{x}$ & $\mathrm{x}$ & & $\begin{array}{l}2,226 \text { trampas } \\
\text { por noche }\end{array}$ \\
\hline & Trampas de caída & $\mathrm{x}$ & $\mathrm{x}$ & $\mathrm{x}$ & & $\begin{array}{l}861 \text { trampas por } \\
\text { noche }\end{array}$ \\
\hline & $\begin{array}{l}\text { Trampas } \\
\text { Tomahawk }\end{array}$ & $\mathrm{x}$ & $\mathrm{x}$ & & & $\begin{array}{l}12 \text { trampas por } \\
\text { noche }\end{array}$ \\
\hline \multirow[t]{2}{*}{$\begin{array}{l}\text { Aves y } \\
\text { mamíferos }\end{array}$} & $\begin{array}{l}\text { Recorridos de } \\
\text { observación }^{(a)}\end{array}$ & $\mathrm{x}$ & $\mathrm{x}$ & $\mathrm{x}$ & $\mathrm{x}$ & $60 \mathrm{~h}$ en $374,3 \mathrm{~km}$ \\
\hline & Cámaras trampa & $\mathrm{x}$ & $\mathrm{x}$ & & $\mathrm{x}$ & $\begin{array}{l}105 \text { trampas por } \\
\text { noche }\end{array}$ \\
\hline
\end{tabular}

(a) Los recorridos de observación incluyen detecciones ad libitum, búsqueda de rastros y de huellas. (b) Tipo de cobertura: $\mathrm{B}$, bosque nativo; $\mathrm{F}$, frailejonal; VE, reforestación con vegetación exótica; $\mathrm{O}$, otras áreas muestreadas: caminos de herradura, humedales, matorrales, pajonales, reforestaciones con aliso, $\mathrm{y}$ viviendas.

Para el registro de pequeños mamíferos no voladores $(<150 \mathrm{~g}$ [11]), durante el 2013, se usaron 42 trampas Sherman $(5 \times 6,4 \times 16,5 \mathrm{~cm}, 7,6 \times 8,9 \times 22,8$ $\mathrm{cm}, 10 \times 11,4 \times 38 \mathrm{~cm}$ ) y 21 trampas de caída (altura $45 \mathrm{~cm}$; diámetro de la base $30 \mathrm{~cm}$ ) en tres tipos de coberturas: bosque, frailejonal y área con vegetación exótica. Las trampas se dispusieron en tres cuadrículas de trampeo de $7 \times 3$, para un total de 63 trampas. El tiempo de muestreo en esta época fue de 17 días no consecutivos, para un esfuerzo de 1,071 trampas por noche. En el 2014 se organizaron cuatro cuadrículas de $7 \times 4$, únicamente en la transición bosque-frailejonal; en cada cuadrícula se distribuyeron entre 20 y 22 trampas Sherman y entre seis y ocho trampas de caída, para un total de 84 trampas Sherman y 28 trampas de caída (total 112 trampas). En esta época, el tiempo de muestreo fue de 18 días no consecutivos, para un esfuerzo de 2016 trampas por noche. 
Para medianos mamíferos (150g-5 kg [11]) se usaron dos trampas Tomahawk $(25 \times 30 \times 81 \mathrm{~cm})$ durante seis días y tres cámaras trampa (Bushnell Trophy Cam 8MP) durante 35 días, con un esfuerzo de 12 y 105 trampas noche respectivamente. Estos métodos fueron usados en bosque, frailejonal y áreas con vegetación exótica. Las cámaras trampa fueron usadas, adicionalmente, en caminos de herradura, por los cuales se realizaron recorridos de observación y búsqueda de rastros. De manera similar que para el muestreo de aves, se examinaron las áreas adyacentes a los caminos desde las 06:00 h hasta las 17:00 h. Finalmente, se realizaron preguntas a pobladores locales acerca de la presencia de algunas especies, con el apoyo de imágenes en guías de campo [23]. Para conocer la representatividad del muestreo y riqueza de especies de ambos grupos, se realizaron análisis mediante el programa Estimates 9,0 [24]. Para aves, se realizaron curvas de acumulación en diferentes coberturas vegetales, donde cada muestra se estableció a partir de 20 registros, de acuerdo con Villareal et al. [1996, 22]; para este análisis se excluyeron datos de coberturas con baja intensidad de muestreo. Se utilizó el estimador no paramétrico Chao 1, que tiene en cuenta las abundancias de las especies. Para mamíferos, la representatividad del muestreo fue obtenida a partir del número de registros por día de muestreo; en este caso, los valores de riqueza esperados se determinaron a partir de los estimadores no paramétricos basados en presencia ausencia, Jack 2 y Michaelis-Menten.

La identificación de las especies se logró mediante literatura especializada [23-28]. Las vocalizaciones fueron identificadas a través de guías sonoras y página web [29, 30]. Para la ordenación taxonómica de aves se siguió a Remsen et al. [31], y para mamíferos, a Solari et al. [32]. Para los nombres comunes se usó el conocimiento popular, pero si no había nombre dado por la gente de la región se usaron los nombres en español $[23,25]$. Con base en el tipo de cobertura vegetal utilizada y la literatura [25, 33], se identificaron especies de aves sensibles a las perturbaciones antrópicas. Para aves y mamíferos se consultaron las categorías de endemismo [25, 32, 34, 35] y amenaza [36-38]. Para aves también se revisó qué especies eran migratorias o acuáticas [39, 40]. Los pequeños mamíferos preservados como especímenes se ingresaron a la colección del Museo de Historia Natural de la Universidad Pedagógica y Tecnológica de Colombia, Uptc.

\section{Resultados y discusión}

En la actualidad, y hasta donde la revisión de la literatura indicó, no se registran estudios que permitan conocer y diagnosticar el estado actual de la avifauna y la mastofauna del páramo de Rabanal. Los listados plasmados en informes y planes de manejo disponibles citan la presencia potencial de algunas especies, pero faltan detalles sobre los métodos utilizados para la recolección de los datos y para las identificaciones $[19,20]$. Por ello, es necesario confirmar su existencia dentro del área para poder tomar decisiones adecuadas para la conservación de los ecosistemas de alta montaña presentes en Rabanal.

En este estudio se confirma la presencia de 57 especies de aves y 14 especies de mamíferos. Para aves, las curvas de acumulación de especies por cobertura vegetal indican una representatividad del muestreo del $86 \%$ para vegetación exótica, $88 \%$ para páramo y $95 \%$ para bosque (figura 2). Para mamíferos, el muestreo tuvo una representatividad entre el $87 \mathrm{y}$ $88 \%$ (tabla 2).

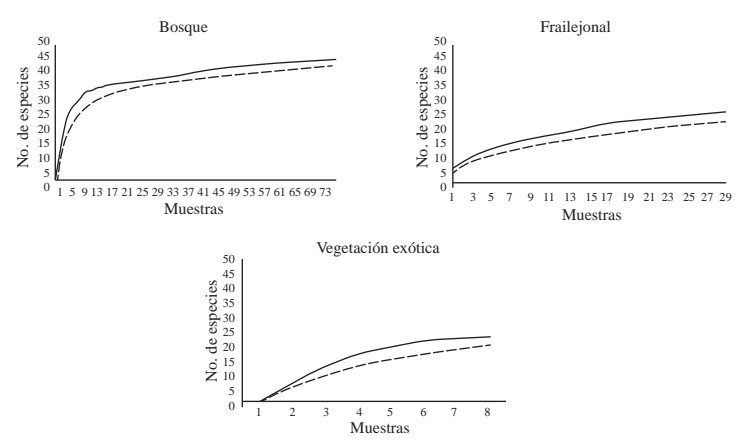

Figura 2. Curvas de acumulación de especies de aves en tres coberturas vegetales en el sector Matanegra, vereda Montoya del municipio de Ventaquemada (Boyacá, Colombia). Línea continua, estimador de Chao 1; línea punteada, especies observadas.

Tabla 2. Representatividad de muestreo de mamíferos en el sector Matanegra (Ventaquemada-Boyacá) con base en los estimadores de riqueza de Michaelis-Menten y Jacknife 2.

\begin{tabular}{lccc}
\hline Grupo & $\begin{array}{c}\text { Especies } \\
\text { observadas }\end{array}$ & MMMean (\%) & Jack 2 Mean (\%) \\
Mamíferos & 14 & 87,5 & 88,4 \\
\hline
\end{tabular}

Esta representatividad es alta, si se compara con los resultados de otros estudios en rangos altitudinales similares. Sin embargo, por ejemplo, la riqueza de aves y mamíferos encontrada en este estudio fue 
un poco más baja que la encontrada en dos zonas protegidas del departamento de Boyacá (tabla 3), la Reserva Ranchería y el Santuario de Fauna y Flora Iguaque [14, 17, 18, 33, 41-43]. Los valores de Rabanal están considerablemente por debajo de los del Parque Nacional Natural Chingaza [10, 12, 16, 44-46], y posiblemente esto se deba a la mayor extensión de Chingaza (tabla 3). En efecto, se espera una relación positiva entre la riqueza de especies y la disponibilidad de ecosistemas naturales [47]. La extensión de muestreos en otras áreas dentro del complejo de páramos Rabanal-Río Bogotá debería incrementar el área y, posiblemente, agregar especies no identificadas durante este trabajo.

Tabla 3. Comparación de los valores de riqueza obtenidos en distintas localidades del altiplano Cundiboyacense en rangos altitudinales similares a los de este trabajo.

\begin{tabular}{lcccc}
\hline Localidad & \multicolumn{2}{c}{$\begin{array}{c}\text { No. de } \\
\text { especies } \\
\text { Aves Mamíferos }\end{array}$} & Área (ha) & $\begin{array}{l}\text { Rango } \\
\text { altitudinal } \\
(\mathrm{m})\end{array}$ \\
\hline $\begin{array}{l}\text { Sector Matanegra } \\
\text { (presente estudio) }\end{array}$ & 56 & 14 & 753 & $3030-3350$ \\
$\begin{array}{l}\text { PNM Ranchería } \\
\text { (a) }\end{array}$ & 60 & 19 & 679 & $2950-3495$ \\
$\begin{array}{l}\text { SFF Iguaque } \\
92^{(b)}\end{array}$ & $13^{(\mathrm{c})}$ & 6,750 & $2700-3250$ \\
$\begin{array}{l}\text { PNN Chingaza } \\
\text { y áreas vecinas }\end{array}$ & 177 & 36 & 76,600 & $2500-3400$ \\
\hline
\end{tabular}

(a) Información recopilada para aves $[14,41]$ y para mamíferos pequeños [17, 18, 42]. (b) [33]. (c) Datos sin corroborar obtenidos del Plan de Manejo SFF Iguaque [43]. (d) Información recopilada para aves $[10,12]$ y para mamíferos [16, 44-45].

Los fragmentos de bosque altoandino, los frailejonales y áreas con vegetación exótica recibieron el mayor esfuerzo de muestreo (tabla 1), y en estas áreas se encontró el mayor número de especies de aves y mamíferos (figura 3); sin embargo, el conjunto de matorrales dispersos entre los pajonales, donde se dedicó un esfuerzo de muestreo bajo, presentaron una riqueza comparable a la de las áreas mencionadas anteriormente (figura 3). En efecto, estos matorrales en distintos estados de sucesión, constituidos por arbustos y arbolitos, son altamente demandados por los animales, ya sea por la oferta de recursos como alimento, sitios para anidación o la disponibilidad de refugio. Así, es probable que estos matorrales hagan parte de corredores que conectan áreas naturales en una matriz de transformación humana [48]. En un escenario de manejo ideal, todas las áreas de Rabanal deberían recuperarse a sus estados originales; sin embargo, este propósito, aunque noble, podría ser de gran dificultad o incluso imposible en algunos casos, debido al avanzado estado de las perturbaciones. Los resultados del presente estudio sugieren que el manejo de áreas nativas y no nativas podría ayudar a mantener la funcionalidad de las comunidades, y en el caso de las áreas de matorral podrían ayudar a acelerar procesos de restauración [49].

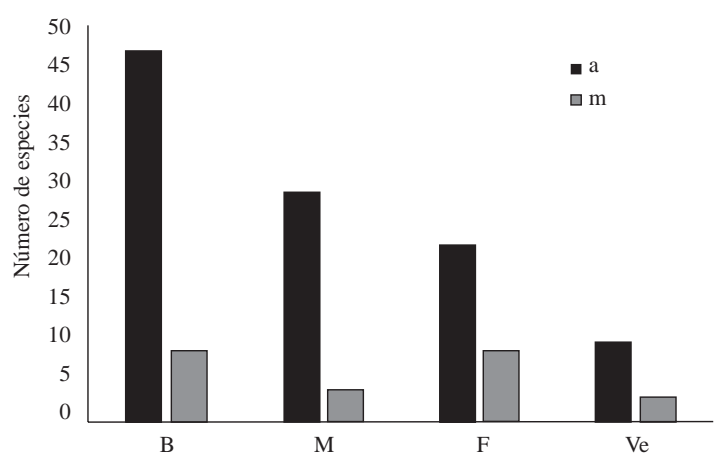

Figura 3. Riqueza de aves (a) y mamíferos (m) en distintas coberturas del sector Matanegra. $\mathrm{B}$, bosque $(\mathrm{a}=$ $42 ; \mathrm{m}=8) ; \mathrm{M}$, matorral $(\mathrm{a}=26 ; \mathrm{m}=4) ; \mathrm{F}$, frailejonal (a $=20 ; \mathrm{m}=8) ; \mathrm{Ve}$, reforestación con vegetación exótica $(\mathrm{a}=9 ; \mathrm{m}=3)$. Nótese el alto número de especies de aves registrado en los matorrales; sitios que tuvieron bajo esfuerzo de muestreo en comparación con el bosque, el frailejonal y el área reforestada con exóticas. Las coberturas restantes, no mostradas por sus bajos valores de riqueza de ambos grupos, corresponden a humedales (a $=4 ; \mathrm{m}=1)$, pajonales $(\mathrm{a}=4 ; \mathrm{m}=3)$, reforestación con aliso $(\mathrm{a}=2 ; \mathrm{m}=3)$, viviendas $(\mathrm{m}=4)$ y caminos de herradura $(m=3)$.

\subsection{Aves}

En el sector Matanegra (Anexo 1) se registraron 24 familias, de las cuales, Thraupidae (9), Trochilidae (7) y Emberizidae (4) presentan el mayor número de especies; nueve taxones con distribución restringida: dos subespecies endémicas y siete especies casi endémicas de Colombia (tabla 4). A su vez, se registraron diez especies que realizan algún tipo de migración, de las cuales, cinco son especies migratorias latitudinales y cinco son migratorias altitudinales [43] (Anexo I). Ninguna de las especies de aves registradas en el sector Matanegra están amenazadas, aunque el colibrí de calzones (Eriocnemis cupreoventris) se reportó años atrás como casi amenazada (NT) [36], y actualmente se considera como de preocupación menor (LC) [37]. 
Tabla 4. Especies de aves y mamíferos con distribución restringida y categoría de amenaza, registrados en el sector Matanegra, del páramo de Rabanal.

\begin{tabular}{|c|c|c|c|}
\hline $\begin{array}{l}\text { Especie } \\
\text { Aves }\end{array}$ & $\begin{array}{l}\text { Nombre } \\
\text { común }\end{array}$ & Endemismo & $\begin{array}{c}\text { Categoría } \\
\text { de amenaza }\end{array}$ \\
\hline Anas andium & Cerceta andina & $\mathrm{CE}$ & LC \\
\hline $\begin{array}{l}\text { Colinus cristatus } \\
\text { (bogotensis) }\end{array}$ & Perdiz & SE & LC \\
\hline $\begin{array}{l}\text { Fulica americana } \\
\text { (columbiana) }\end{array}$ & Polla de agua & SE & LC \\
\hline Gallinago nobilis & Caica & $\mathrm{CE}$ & $\mathrm{LC}$ \\
\hline $\begin{array}{l}\text { Eriocnemis } \\
\text { cupreoventris }\end{array}$ & $\begin{array}{l}\text { Colibrí de } \\
\text { calzones }\end{array}$ & $\mathrm{CE}$ & LC \\
\hline Conirostrum rufum & Picocono rufo & $\mathrm{CE}$ & $\mathrm{LC}$ \\
\hline $\begin{array}{l}\text { Atlapetes } \\
\text { pallidinucha }\end{array}$ & Gorrión de bosque & $\mathrm{CE}$ & LC \\
\hline Myioborus ornatus & $\begin{array}{l}\text { Candelita } \\
\text { frentidorada }\end{array}$ & $\mathrm{CE}$ & LC \\
\hline Sporagra spinescens & Chisga de páramo & $\mathrm{CE}$ & LC \\
\hline \multicolumn{4}{|l|}{ Mamíferos } \\
\hline Cryptotis thomasi & Musaraña & E & $\mathrm{LC}$ \\
\hline $\begin{array}{l}\text { Thomasomys } \\
\text { niveipes }\end{array}$ & Ratón & $\mathrm{E}$ & LC \\
\hline $\begin{array}{l}\text { Cuniculus } \\
\text { taczanowskii }\end{array}$ & Tinajo & & NT \\
\hline Nasuella olivacea & Guache, runcho & & DD \\
\hline \multicolumn{4}{|c|}{$\begin{array}{l}\text { Endemismos de aves }[25,34] \text { y de mamíferos }[32,35] \text {, en categorías } \\
\text { de amenaza para a aves [36-38] y para mamíferos }[32,50-52] \text {. En pa- } \\
\text { réntesis subespecies. CE, Casi Endémica; SE, Subespecie Endémica; } \\
\text { E, Endémica; LC, preocupación menor; NT, Casi Amenazada; DD, } \\
\text { Datos insuficientes. }\end{array}$} \\
\hline
\end{tabular}

El sector Matanegra, presenta nueve especies de aves con distribución restringida, característica típica de las comunidades de los Andes Orientales de Colombia, los cuales han sido definidos como un área de endemismo de aves EBA [53]. Estas especies deberían ser consideradas una prioridad de conservación, ya que toda la responsabilidad, o gran parte de ella, recae sobre Colombia [54].

El $57 \%$ de las especies de aves son reconocidas como sensibles a la perturbación antrópica (Anexo 1), y cinco especies son altamente sensibles; estas especies, al igual que las especies endémicas deberían ser definidas como prioridades de conservación específicas [33]. El $43 \%$ restante corresponde a especies que pueden emplear una amplia variedad de hábitats, como la mirla común (Turdus fuscater), que fue registrada en todos los hábitats de estudio.

En este estudio se confirma la presencia de 20 de las 57 especies de aves registradas en informes y planes de manejo para el complejo Rabanal-Río Bogotá $[9,19,20]$. A su vez, este trabajo presenta 35 nuevos registros para el área de estudio (Anexo 1), siendo especies típicas de páramo y bosque altoandino de la cordillera Oriental colombiana [55].
Esta investigación permitió establecer la presencia de cuatro especies de aves acuáticas estrictas y una no estricta [40] (Anexo 1). En Laguna Verde, durante el mes de marzo de 2014, se registró un adulto de la polla de agua (Fulica americana) con tres polluelos; por otro lado, en febrero del mismo año se registraron un macho y tres hembras del pato paramuno (Anas discors) en el mismo sitio. Durante todos los meses de muestreo se registraron dos individuos de pato de páramo (A. andium) tanto en Laguna Verde como en Laguna Cristal, y desde agosto de 2013 hasta marzo de 2014 se encontró continuamente un individuo de chorlo de patas amarillas (Tringa melanoleuca), lo que sugiere la permanencia de la especie durante el verano norteño, y puede tratarse de un individuo juvenil, como ha sido mencionado para la Sabana de Bogotá [25]. En enero de 2014 se identificó el mayor número de individuos de aves acuáticas en Laguna Cristal, con una bandada de 13 individuos de pato páramo (A. andium) y 10 individuos de chorlo de patas amarillas (T. melanoleuca); sin embargo, en febrero no se observaron estas especies debido a la desecación del humedal. Por otro lado, la caica (Gallinago nobilis) y el sotorrey de pajonal (Cistothorus platensis) se registraron continuamente en las áreas de pajonal y frailejonal, y este último fue la única especie acuática no estricta.

Durante los muestreos de aves se registraron datos puntuales de alimentación de algunas especies. El siote (Turdus fuscater) y el clarinero (Anisognathus igniventris) consumieron frutos de Miconia cataractae, similar a lo descrito para Cundinamarca [49], y otra especie de Miconia. Individuos del carbonero común (Diglossa humeralis) se encontraron forrajeando solitarios flores de $M$. cataractae, comportamiento observado también en un bosque altoandino del departamento de Cundinamarca [56]. Mientras que el picocono rufo (C. rufum) conformó bandadas mixtas y forrajeó en flores de Diplostephium sp., y el atrapamoscas (Mecocerculus leucoprhys), el trepadorcito (Margarornis squamiger), la reinita coroninegra (Myiothlypis nigrocristata), la candelita frentidorada (M. ornatus) y el hemispingo superciliado (Hemispingus superciliaris), especies insectívoras, se alimentaron en el follaje de Diplostephium sp. En el mes de marzo se registró a $P$. goudotiana en estado de floración con visitas de los colibríes Eriocnemis vestita, Lesbia victoriae, Pterophanes cyanopterus, y del carbonero común (D. humeralis). 
En el mes de septiembre de 2013 se registró actividad reproductiva para tres especies de aves. Se evidenció un individuo de carbonero brillante ( $\mathrm{Di}$ glossa lafresnayii) con parche de cría, y se hallaron nidos de colibrí (Colibri coruscans) y carbonero común (D. humeralis), lo cual coincide con el principal periodo reproductivo de estas especies en el parque municipal Ranchería, el cual fue entre abril y agosto; este período también coincide con el de mayor floración de las especies ornitófilas [14]. En marzo se registró un polluelo de siote ( $T$. fuscater) siendo alimentado. Adicionalmente, se presume anidación del águila pechinegra (Geranoaetus melanoleucus) en febrero de 2014, ya que se registró un juvenil y un adulto; el adulto mostró un comportamiento de defensa similar a lo descrito en la Patagonia Argentina [57].

\subsection{Mamíferos}

Para el sector Matanegra se registró un total de 11 familias y siete órdenes, con una especie por familia, a excepción de Cricetidae, que tuvo cuatro. En este estudio se confirmó la presencia de ocho de las nueve especies de mamíferos medianos reportados en informes y planes de manejo para el complejo Rabanal-Río Bogotá $[9,19,20]$. Dos de estas están bajo algún riesgo de amenaza, según la Unión Internacional para la Conservación de la Naturaleza (UICN) (tabla 4). De las seis especies de pequeños mamíferos registrados, cuatro no habían sido documentadas para el páramo de Rabanal: Thomasomys niveipes, Cryptotis thomasi, Microryzomys minutus y Oligoryzomys fulvescens (Anexo 2). De estas, las dos primeras son catalogadas como endémicas para el altiplano Cundiboyacense [35, 58] (tabla 4).

El único mamífero volador registrado, el murciélago orejón andino, Histiotus montanus, fue identificado a través de rasgos morfológicos externos, y verificado a través de guía de campo [23] mientras se encontraba perchado en el techo de una vivienda abandonada. Se recomienda usar redes de niebla durante la noche para verificar la presencia de las especies mencionadas en informes y planes de manejo [19, 20].

La musaraña (Cryptotis thomasi) presentó comportamiento caníbal; esto ocurrió en dos ocasiones en que se encontraron dos musarañas en las trampas de caída, una de ellas muerta con algunas partes del cuerpo consumidas, mientras que la otra se encontraba viva y muy activa; se ha sugerido que por el encerramiento, la consecuente falta de alimento y su alto metabolismo, las musarañas adoptan esta conducta $[23,59]$.

Se identificó el curí silvestre (Cavia aperea) [23, 60] a partir de un espécimen encontrado muerto, presumiblemente por perros ferales que fueron registrados en una cámara trampa la noche anterior. Por otro lado, en el borde bosque-frailejonal se observó un individuo de T. niveipes junto a un individuo de C. aperea, a las 08:00 h. Aunque hay reportes que indican que individuos del género Thomasomys y del curí silvestre comparten hábitats similares [45], no se encontró información que explique este comportamiento, pero el forrajeo en grupo podría mejorar la sensación de seguridad de los organismos [61].

Individuos de comadreja (Mustela frenata) fueron capturados en trampas Sherman en frailejonal, probablemente atraídos por el olor de los ratones capturados $[16,44]$. Heces de guache (N. olivacea), encontradas en bordes de bosque, frailejonal, pajonal y caminos de herradura, contenían restos de cutícula esclerotizada de insectos o semillas de Rubus sp., como ha sido reportado para Chingaza [16]. Además, fue común encontrar señales de su forrajeo (hozaderos) en bordes de bosque y a la orilla de caminos de herradura.

En imágenes grabadas en cámaras trampa, el tinajo (C. taczanowskii) apareció consumiendo hojas de Rubus sp., mientras que en otras aparece compartiendo hábitat con $D$. pernigra y T. niveipes, pero en distintas horas de la noche. Por otro lado, el armadillo (Dasypus novemcinctus) fue registrado a partir de huellas en caminos de herradura y a partir de un caparazón encontrado en una vivienda y usado como contenedor; esta es una de las especies más usadas para consumo humano en los Andes [11, 61]. Una pareja de zorro gatuno (Urocyon cinereoargenteus) fue registrada en las cámaras trampa al amanecer, en el borde bosque-frailejonal, donde es común encontrar senderos y rastros de curíes y roedores, animales que pueden ser parte de su dieta [44]. Finalmente, el conejo sabanero (Sylvilagus brasiliensis) fue registrado a partir de heces en el pajonal y por observación de un individuo en el borde bosque-pastizal; la presencia en estos sitios es debida a su preferencia por las hierbas, particularmente de la familia Poaceae [44, 62]. 


\subsection{Ausencia de especies}

La avifauna y mastofauna registradas aquí representan menos de la mitad de la fauna de ecosistemas de altitud similar en la cordillera Oriental [10, 12, 14, 16-18, 33, 41-46], y, probablemente, representa un subconjunto de la fauna regional que persiste en ecosistemas naturales fragmentados. Según pobladores locales, en el pasado eran comunes en Rabanal especies de frugívoros grandes como el tucán esmeralda (Aulacorhynchus prasinus), la pava de monte (Penelope montagnii) y el periquito aliamarillo (Pyrrhura calliptera) [20], o especies de gran porte como el oso de anteojos (Tremarctos ornatus), el venado (Odocoileus virginianus, Mazama sp.), el león de monte (Puma concolor) y otras especies de felinos (Leopardus spp.). La ausencia de estas especies en el sector muestreado parece relacionarse con la historia de profunda intervención antrópica a la que ha sido sometido el complejo de páramos de Rabanal [19, 20]. Por lo tanto, se sugiere que debido a la reducción de los bosques a islas-fragmentos y a las amenazas a los hábitats de páramo, es necesario concentrar el esfuerzo en su recuperación a través de la restauración, recuperación o con el uso de estrategias de reconciliación ecológica que permitan integrar a la comunidad local [63].

\section{Conclusiones}

El sector Matanegra en Rabanal tiene un menor número de aves y mamíferos que el presentado para áreas protegidas de Boyacá y Cundinamarca a alturas similares. El menor número de especies puede relacionarse con la menor área de muestreo incluida en el estudio, pero puede deberse también a la desaparición local de fauna. La presencia de especies con distribución restringida y de especies con amenaza de extinción apoya la importancia de la declaratoria de este páramo como área protegida [64], y justifica el desarrollo de acciones de conservación que garanticen la permanencia de las especies en el área [22]. La necesidad de preservar la integridad ecosistémica que permita mantener a Rabanal como fuente de agua también debería justificar la conservación de la fauna nativa de la zona. Entre estas acciones se sugiere la puesta en marcha de procesos pedagógicos ambientales con la comunidad local y el aumento de estudios de investigación que permitan entender las relaciones ecológicas y el efecto de las perturbaciones humanas sobre el comportamiento de las especies. Esto es porque las amenazas para la biodiversidad en la zona se relacionan con las actividades humanas y estas ya han cobrado la existencia local de varias especies. Por lo anterior, solo si las comunidades locales participan de manera activa en la preservación de la biodiversidad que las rodea, será posible conservar las aves y mamíferos que observamos hoy, para las generaciones futuras.

\section{Agradecimientos}

Este estudio hace parte del proyecto “ ¿Las modificaciones al paisaje afectan la coexistencia de aves y mamíferos en un área de transición bosque altoandinopáramo en Boyacá?", financiado por el convenio entre la Universidad Pedagógica y Tecnológica de Colombia (UPTC) y Colciencias, a través de la convocatoria de becas No. 566 (Joven Investigador 20132014). Expresamos nuestros agradecimientos al grupo de investigación Sistemática Biológica (SisBio), a su líder, María Eugenia Morales-Puentes, y al equipo de trabajo de campo. A Naisla Manrique y Juan Carlos Zabala, por la determinación de material vegetal, y Pablo Gil, por la elaboración del mapa. A don Cristobal Reyes y familia, quienes nos brindaron su colaboración con la estadía. A Juan Pablo Gómez y Eduardo Gallo-Cajiao, quienes aportaron significativamente con comentarios y sugerencias a versiones preliminares del manuscrito, e igualmente, a dos revisores anónimos.

\section{Referencias}

[1] A. Etter, and W. van Wyngaarden, "Patterns of landscape transformation in Colombia, with emphasis in the Andean Region", Ambio, vol. 29, no. 7, pp. 432-439. 2000.

[2] J. O. Rangel-Ch., "Visión integradora sobre la región del páramo". En J.O. Rangel-Ch. (ed.): Colombia Diversidad Biótica III. La región de vida paramuna, pp. 814-836. Bogotá: Universidad Nacional de Colombia. Instituto de Ciencias Naturales, 2000.

[3] D. Ojeda, J. Pinto, M. C. Cardona, M. Cuellar, S. Cruz, L. S. de la Torre, J. Castañeda, C. R. Barrera, Y. González, and J. C. Alarcón, "Ecosistemas", En P. Leyva: El medio ambiente en Colombia. Bogotá: IDEAM, 2001. 
[4] P. Foster, "The potential negative impacts of global climate change on tropical montane cloud forests". Earth-Science Reviews, vol. 55, pp. 73-106, 2001.

[5] D. Armenteras, F. Gast, and H. Villareal, "Andean forest fragmentation and the representativeness of protected natural areas in the eastern Andes, Colombia", Biol. Conserv, vol. 113, pp. 245-256. 2003.

[6] T. van der Hammen, "La dinámica del medio ambiente en la alta montaña colombiana: historia, cambio global y biodiversidad", En J. A. Lozano, and J. D. Pabón. (eds.): Memorias del Seminario Taller sobre Alta Montaña Colombiana, pp. 11-15. Bogotá: Academia Colombiana de Ciencias Exactas, Físicas y Naturales. Colección Memorias, no. 3, 1995,.

[7] C. Castaño-Uribe, R. Carrillo, and F. Salazar, Sistema de Información Ambiental de Colombia Tomo III. Perfil del estado de los recursos naturales y del medio ambiente en Colombia 2001, Bogotá: IDEAM. Ministerio del Medio Ambiente, 2002.

[8] P. A. Verweij, K. Kok, and P. E. Budde, "Aspectos de la transformación del páramo por el hombre", En T. Van der Hammen \& A.G. Dos Santos (eds.): Estudios de ecosistemas tropoandinos, Volumen 5. La Cordillera Central Colombiana. Transecto Parque Los Nevados . Berlín-Stuttgart, 2003.

[9] M. Morales, J. Otero, T. van der Hammen, A. Torres, C. Cadena, C. Pedraza, N. Rodríguez, C. Franco, J. C. Betancourth, E. Olaya, E. Posada, and L. Cárdenas, Atlas de páramos de Colombia, Bogotá: Instituto de Investigación de Recursos Biológicos Alexander von Humboldt, 2007.

[10] F. G. Stiles, and L. Rosselli, "Inventario de las aves de un bosque altoandino: comparación de dos métodos", Caldasia, vol. 20, pp. 29-43, Mayo 1998.

[11] F. Sánchez, P. Sánchez-Palomino, and P. Cadena, "Inventario de mamíferos en un bosque de los Andes centrales de Colombia", Caldasia, vol. 2 (1), pp. 291-309. 2004.

[12] G. I. Andrade, M. L. Rosas, and A. Repizzo, "Notas preliminares sobre la avifauna y la integridad biológica de Carpanta”, En G.I. Andrade (ed.): "Carpanta selva nublada y páramo”. Bogotá: Fundación Natura, pp. 207-228, 1993.

[13] R. Salamanca, J. Botía-Becerra, and A. ArdilaVega, Aves del Páramo de Siscunsí. Sogamoso: Asociación para la Investigación y Conservación de la Vida Silvestre Neotropical, 2008.

[14] L. Rosero, D. L. Tolosa, D. A. León, C. L. Ortiz, A. C. Umba, and A. I. Prada, "Evaluación Ecológica de las aves de los estratos bajos del Parque”, En L. Rosero (ed.): "Estudios Ecológicos en el Parque Natural Municipal Ranchería. Un aporte a su conservación”, pp. 135-170. Tunja: Universidad Pedagógica y Tecnológica de Colombia \& Corporación Autónoma Regional de Boyacá, 2010.

[15] L. A. Meneses, and Y. Herrera-Martínez, "Estudio preliminar de la avifauna asociada a parches de Polylepis quadrijuga (Rosaceae) del páramo de la Rusia, Duitama (BoyacáColombia)", Luna Azul, vol. 36, pp. 40-54, enero 2013.

[16] H. López-Arévalo, and O. Montenegro-Díaz. "Mamíferos no voladores de Carpanta", En G.I. Andrade (ed.): "Carpanta selva nublada y páramo”, pp. 165-187. Bogotá: Fundación Natura, 1993.

[17] J. Cepeda, A. Hernández, E. Muñoz, and A. Vianchá. "Pequeños mamíferos no voladores" En L. Rosero. (ed.): "Estudios Ecológicos en el Parque Natural Municipal Ranchería. Un aporte a su conservación”. Tunja Universidad Pedagógica y Tecnológica de Colombia \& Corporación Autónoma Regional de Boyacá, 2010.

[18] G. Ramírez, and M. Pardo, "Composición, dieta y estructura trófica de la comunidad de murciélagos presente en el área de influencia del Parque". En L. Rosero (ed.): "Estudios Ecológicos en el Parque Natural Municipal Ranchería. Un aporte a su conservación”. Tunja: Universidad Pedagógica y Tecnológica de Colombia \& Corporación Autónoma Regional de Boyacá, 2010.

[19] Instituto de Investigación de Recursos Biológicos Alexander von Humboldt (IAvH), Corporación Autónoma Regional de Cundinamarca 
(CAR), Corporación Autónoma Regional de Boyacá (Corpoboyacá), Corporación Autónoma de Chivor (Corpochivor), "Estudio sobre el estado actual del Macizo del Páramo de Rabanal”, Bogotá, Inf. Tec, No. 07-06-263-048 (000404), mayo de 2008.

[20] Ministerio de Medio Ambiente, Corpochivor, Corpboyacá, CAR. "Plan de manejo y uso sostenible del Páramo de Rabanal”, BoyacáCundinamarca, Inf. Tec. Programa Ambiental Credito Bid 774 oc/Coconvenio no. 00297. Fonade-Convenio No. 000294, 2001.

[21] C. J. Ralph, G. R. Geupel, P. Pyle, T. E. Martin, D. F. DeSante, and M. Borja, Manual de métodos de campo para el monitoreo de aves terrestres, Albany: U.S. Department of Agriculture, Forest Service, Pacific Southwest Research Station, 1996.

[22] H. Villareal, M. Álvarez, S. Córdoba, F. Escobar, G. Fagua, F. Gast, H. Mendoza, Ospina M., and A. M. Umaña, Manual de métodos para el desarrollo de inventarios de biodiversidad, Bogotá: Instituto de Investigación de Recursos Biológicos Alexander von Humboldt, 2006.

[23] D. Tirira, Guía de campo de los mamíferos del Ecuador, publicación especial sobre mamíferos de Ecuador 6, Quito: Ediciones Murciélago Blanco, 2007.

[24] R.K. Colwell, Estimates: Statistical estimation of species richness and shared species from samples, Version 9.1.0 [online]. USA: Univesidad de Connecticut, 2013. Disponible en: http://purl.oclc.org/estimates

[25] Asociación Bogotana de Ornitología, Aves de la Sabana de Bogotá, guía de campo, Bogotá: Asociación Bogotana de Ornitología, Corporación Autónoma Regional de Cundinamarca, 2000.

[26] S. L Hilty, and W. L. Brown, A guide to the birds of Colombia. Princeton: Princeton University Press, 1986.

[27] L. Emmons, and F. Feer, Mamíferos de los bosques húmedos de América Tropical, una guía de campo, Santa Cruz de la Sierra: Editorial FAN Lera, edición en español, 1999.

[28] A. L. Morales-Jiménez, F. Sánchez, K. Poveda, and A. Cadena, Mamíferos terrestres y vola- dores de Colombia, Guía de campo, Bogotá, 2004.

[29] M. Álvarez-Rebolledo, V. Caro, O. Laverde, and A. Cuervo, Guía sonora de la aves de los Andes colombianos, Villa de Leyva: Instituto de Investigación en Recursos Biológicos Alexander von Humboldt, 2007.

[30] B. Planqué, W. P. Vellinga, S. Pieterse, and J. J. "Xeno-Canto. Compartiendo cantos de aves de todo el mundo" [online]. Xeno-Canto Foundation, 2014. Disponible en: http://www.xenocanto.org/

[31] J. V. Remsen, J. I. Areta, C. D. Cadena, A. Jaramillo, M. Nores, J. F. Pacheco, J. Pérez-Emán, M. B. Robbins, F. G. Stiles, D. F. Stotz, and K. J. Zimmer, A classification of the bird species of South America [on line]. American Ornithologists' Union, Version 18 de febrero de 2015. Disponible en: http://www.museum.lsu.edu/ ${ }^{\sim}$ Remsen/SACC Baseline.html

[32] S. Solari, Y. Muñoz-Saba, J. V. RodríguezMahecha, Thomas R. Defler, H. E. RamírezChaves, and F. Trujillo, "Riqueza, endemismo y conservación de los mamíferos de Colombia", Mastozoología Neotropical, vol. 20 (2), pp. 301-365, 2013.

[33] S. Córdoba-Córdoba, and M. A. EcheverryGalvis, "Diversidad de aves de los bosques mixtos y de roble del Santuario de Flora y Fauna de Iguaque, Boyacá", En C. Solano, and N. Vargas (eds): Memorias del primer seminario internacional de Roble y ecosistemas asociados, pp. 119-128. Bogotá: Fundación Natura Colombia, 2006.

[34] S. Chaparro-Herrera, M. A. Echeverry-Galvis, S. Córdoba-Córdoba, and A. Sua-Becerra, "Listado actualizado de las aves endémicas y casiendémicas de Colombia”, Biota Colombiana, no. 2, vol. 14, pp. 235-272, julio-diciembre, 2013.

[35] A. L. Gardner, Mammals of South America, Volume 1. Marsupials, Xernarthrans, Shrews, and Bats. Chicago and London: The University of Chicago Press, 2008.

[36] L. M. Renjifo, A. Franco, J. Amaya-Espinel, G. Kattan, and B. López-Lanus Eds, Libro Rojo 
de Aves de Colombia. Serie Libros Rojos de Especies Amenazadas de Colombia, Bogotá: Instituto de Investigación de Recursos biológicos Alexander von Humboldt y Ministerio del Medio Ambiente, 2002.

[37] L. M. Renjifo, M. F. Gómez, J. VelásquezTibatá, A. M. Amaya-Villareal, G. H. Kattan, J. D. Amaya-Espinel, and J. Burbano-Giron, $L i-$ bro Rojo de Aves de Colombia, Volumen 1: bosques húmedos de los Andes y la Costa Pacífica, Bogotá, D.C: Editorial Pontificia Universidad Javeriana e Instituto Alexander von Humboldt, 2014.

[38] Bird Life International, Red List of Threatened Species [online], International Union for Conservation of Nature and Natural Resources, 2013. Disponible en: www.iucnredlist.org.

[39] L. G. Naranjo, D. Amaya, D. Eusse-González, and Y. Cifuentes-Sarmiento (eds.), Guía de las especies migratorias de la biodiversidad en Colombia, Aves, vol. 1, Ministerio de Ambiente y Desarrollo Sostenible. WWF Colombia, Bogotá, D. C., 2012.

[40] C. Ruiz-Guerra, Listado de aves acuáticas de Colombia, Cali: Asociación Calidris, 2012.

[41] D. L. Toloza-Moreno, D. A. León-Camargo, and L. Rosero-Lasprilla, "El ciclo anual de una comunidad de colibríes (Trochilidae) en bosques altoandinos intactos y paramizados en la cordillera Oriental de Colombia”, Ornitología Colombiana, vol. 14, pp. 28-47, 2014.

[42] A. P. Vianchá, J. Y. Cepeda-Gómez, E. C. Muñoz, A. M. Hernández, and L. Rosero, "Mamíferos pequeños no voladores del Parque Natural Municipal Ranchería, Paipa, Boyacá, Colombia”, Biodiversidad Neotropical, vol. 2 , no. 1, pp. 37-44, 2012.

[43] Parques Nacionales Naturales de Colombia, "Plan de manejo Santuario de Flora y Fauna Iguaque", Ministerio de Ambiente y Desarrollo Territorial, 2006.

[44] J. Pérez-Torres, and J. E. Correa, "Anotaciones sobre los Artiodactyla, Carnivora, y Perissodactyla del Parque Nacional Natural Chingaza (I)", Universitas Scientarium, vol. 2, no. 2, pp. 25-41, julio-diciembre 1995.

[45] J. Pérez-Torres, and J. E. Correa, "Anotaciones sobre los Lagomorpha y Rodentia del Parque
Nacional Natural Chingaza (III)", Universitas Scientarium, vol. 4, no. 1, pp. 31-50, enerojunio 1997.

[46] J. E. Correa, and J. Pérez-Torres, "Anotaciones sobre los Chiroptera, Edentata, Insectívora, Marsupialia y Primates del Parque Nacional Natural Chingaza (II)", Universitas Scientarium, vol. 3, no. 1-2, pp. 25-39, julio-diciembre 1996.

[47] K. J. Gaston, and T. M. Blackburn, Pattern and process in macroecology, Blackwell Science, Oxford, 2000.

[48] O. Vargas (ed.), Guía metodológica para la restauración ecológica del bosque altoandino, Bogota: Universidad Nacional de Colombia, 2007.

[49] P. Velasco-Linares, and O. Vargas, "La dispersión por aves y la restauración de los ecosistemas Altoandinos", en O. Vargas (ed.): Estrategias para la restauración ecológica del bosque altoandino. El caso de la Reserva Forestal Municipal de Cogua, Cundinamarca, pp. 217-228. Bogotá: Universidad Nacional de Colombia, 2008.

[50] F. Reid, and K. Helgen, Nasuella olivacea, Red List of Threatened Species [online], International Union for Conservation of Nature and Natural Resources, 2013. Disponible en: www.iucnredlist.org

[51] D. Tirira, C. Boada, and J. Vargas, Nasuella olivacea, Red List of Threatened Species [online], International Union for Conservation of Nature and Natural Resources. Disponible en: www.iucnredlist.org

[52] S. A. Balaguera-Reina, A. Cepeda, D. ZárrateCharry, and J. F. González-Maya, "The state of knowledge of Western Mountain Coati Nasuella olivacea in Colombia, and extent of occurrence in the Northern Andes", Small carnivore conservation, no. 41 (special issue: The Americas), pp. 35-40, 2009.

[53] Bird Life International. Country profile: Colombia. [online] International Union for Conservation of Nature and Natural Resources, 2015. Disponible en: http://www.birdlife.org/datazone/country/ colombia 
[54] G. Stiles, "Aves endémicas de Colombia", En M. E. Chaves, and N. Arango (eds.): Informe nacional sobre el estado de la biodiversidad en Colombia ( vol. I), pp. 378-385. Bogotá: Instituto de Investigación de Recursos Biológicos Alexander von Humboldt, 1998.

[55] A. C. Delgado, and O. Rangel. Aves. En J.O. Rangel-Ch. (ed.): Colombia Diversidad Biótica III, pp. 629-644. Bogotá: Universidad Nacional de Colombia. Instituto de Ciencias Naturales, 2000.

[56] S. V. Rojas, "Ecología de la comunidad de pinchaflores (Aves: Diglossa y Diglossopis) en un bosque altoandino", Tesis de Maestría, Departamento de Biología, Universidad Nacional de Colombia, Bogotá, 2005.

[57] M. D. Saggese, and E. R. De Lucca, "Biología reproductiva del águila mora (Geranoaetus melanoleucus) en la Patagonia sur, Argentina", Hornero, vol. 16, no. 02, pp. 077-084, Diciembre 2001.

[58] P. Rivas, and M. Gómez-Laverde, Thomasomys niveipes, Red List of Threatened Species [online], International Union for Conservation of Nature and Natural Resources, 2013. Disponible en: www.iucnredlist.org

[59] J. Choate, J. Jones, and C. Jones, Handbook of mammals of the South-Central states, Baton Rouge and London: Louisiana State University Press, 1994.

[60] J. Dunnum, and J. Salazar-Bravo, "Molecular systematics, taxonomy and biogeography of the genus Cavia (Rodentia: Caviidae)", Journal of Zoological Systematics and Evolutionary Research, vol. 28, pp. 376-388, 2010.

[61] W. H. Buskirk. "Social systems in a tropical forest avifauna", American Naturalist, 110, pp. 293-310, 1976.

[62] F. Sánchez, and M. Alvear, "Comentarios sobre el uso de hábitat, dieta y conocimiento popular de los mamíferos en un bosque andino de Caldas, Colombia", Boletín Científico, Museo de Historia Natural, Universidad de Caldas, vol. 7, pp. 121-144, 2003.

[63] M. L. Rosenzweig, "Win-win ecology: how Earth's species can survive in the midst of human enterprise", Oxford, Reino Unido: Oxford University Press, 2003.
[64] M. Urdaneta, "Propuesta para la declaratoria de un área protegida en el páramo de Rabanal", Informe de avance. Bogotá: Instituto de Investigación de Recursos Biológicos Alexander von Humboldt: agosto, 2008.

\section{Anexo 1}

Lista de aves registradas en el Sector Matanegra, Páramo de Rabanal. Se sigue la propuesta taxonómica de [31]. Nombres comunes con base en [25]. Especies sensibles de acuerdo con ABO [26, 33] y observaciones realizadas en este estudio. Definición de especies migratorias con base en [39]. Método de registro: $\mathrm{RO}$, recorridos de observación; $\mathrm{PC}$, puntos de conteo; R, captura con redes de niebla; $\mathrm{CT}$, cámaras trampa. Cobertura: B, bosque; F, frailejonal; $\mathrm{M}$, matorral; P, Pajonal; VE, reforestación con vegetación exótica; A, reforestación con aliso; $\mathrm{H}$, humedal. Especies Sensibles: S, sensible; AS, altamente sensible. Categoría migratoria: lat, migración latitudinal; ir, invernante reproductivo; inr, invernante no reproductivo; alt, migración altitudinal. (a) Especies no registradas previamente para la zona por [20].

\section{Anexo 2}

Lista de mamíferos pequeños y medianos del sector Matanegra, Páramo de Rabanal. Se señala el método de registro, y el (los) hábitat(s) donde se registraron las especies. Se sigue la propuesta taxonómica de [32]. Nombres comunes con base en conocimiento popular local y [23]. Método de registro: CT, cámara trampa; T, Tomahawk; TC, trampa de caída; S, Sherman; R, Rastros; E, encuestas; O, Observación directa. Cobertura: B, bosque; F, frailejonal; $\mathrm{CH}$, camino de herradura; $\mathrm{M}$, matorral; $\mathrm{V}$, vivienda; $\mathrm{P}$, Pajonal; VE, reforestación con vegetación exótica; A, reforestación con aliso; H, humedal. (a) Especies no registradas previamente para la zona por [20]. 
Tabla. Anexo 1.

\begin{tabular}{|c|c|c|c|c|c|c|}
\hline Familia & Especie & $\begin{array}{l}\text { Nombre } \\
\text { común }\end{array}$ & $\begin{array}{l}\text { Método de } \\
\text { registro }\end{array}$ & Cobertura & $\begin{array}{l}\text { Especies } \\
\text { sensibles }\end{array}$ & $\begin{array}{l}\text { Categoría } \\
\text { migratoria }\end{array}$ \\
\hline ANATIDAE & Anas andium $^{(a)}$ & Cerceta Andina & $\mathrm{RO}, \mathrm{OA}$ & $\mathrm{H}$ & & \\
\hline ANATIDAE & Anas discors $^{(a)}$ & Pato Canadiense & $\mathrm{OA}$ & $\mathrm{H}$ & & lat, ir \\
\hline ODOTOPHORIDAE & Colinus cristatus & Perdiz & RO & M & & \\
\hline CATHARTIDAE & Coragyps atratus & Chulo & RO & B & & \\
\hline ACCIPITRIDAE & Elanus leucurus & Gavilán Bailarín & RO & $\mathrm{P}$ & & \\
\hline ACCIPITRIDAE & Geranoaetus melanoleucus & Águila Pechinegra & RO & $\mathrm{F}, \mathrm{B}$ & & \\
\hline RALLIDAE & Fulica americana ${ }^{(a)}$ & Polla de Agua & RO & $\mathrm{H}$ & & \\
\hline SCOLOPACIDAE & Gallinago nobilis & Caica & RO & $\mathrm{F}$ & & \\
\hline SCOLOPACIDAE & Tringa melanoleuca ${ }^{(a)}$ & Chorlo de Patas Amarillas & RO & $\mathrm{H}$ & & lat, inr \\
\hline COLUMBIDAE & Patagioenas fasciata & Paloma Collareja & $\mathrm{RO}, \mathrm{PC}$ & $\mathrm{B}, \mathrm{VE}$ & $\mathrm{S}$ & alt \\
\hline COLUMBIDAE & Zenaida auriculata & Torcaza & $\mathrm{RO}, \mathrm{PC}$ & $\mathrm{B}, \mathrm{VE}$ & & \\
\hline STRIGIDAE & Megascops choliba & Currucutú & RO & M & $\mathrm{S}$ & \\
\hline STRIGIDAE & Megascops albogularis $(a)$ & Lechuzita Barbiblanca & RO & M & AS & \\
\hline STRIGIDAE & Glaucidium jardinii $^{(a)}$ & Buhito & $\mathrm{RO}, \mathrm{PC}$ & $\mathrm{B}, \mathrm{F}, \mathrm{VE}$ & $\mathrm{S}$ & \\
\hline CAPRIMULGIDAE & Systellura longirostris ${ }^{(a)}$ & Gallinaciega & $\mathrm{RO}, \mathrm{PC}$ & $\mathrm{B}, \mathrm{M}$ & & \\
\hline APODIDAE & Streptoprocne zonaris ${ }^{(a)}$ & Vencejo & RO & $\mathrm{B}, \mathrm{M}$ & & \\
\hline TROCHILIDAE & Colibri coruscans & Colibrí, Tomineja & $\mathrm{PC}, \mathrm{RO}$ & $\mathrm{B}, \mathrm{M}$ & & alt \\
\hline TROCHILIDAE & Lesbia victoriae $^{(a)}$ & Colibrí de cola larga & $\mathrm{RO}, \mathrm{PC}, \mathrm{CT}$ & $\mathrm{B}, \mathrm{F}$ & $\mathrm{S}$ & alt \\
\hline TROCHILIDAE & Metallura tyrianthina & Tomineja Gragantona & $\mathrm{RO}, \mathrm{PC}$ & $\mathrm{B}, \mathrm{M}, \mathrm{F}$ & & alt \\
\hline TROCHILIDAE & Eriocnemis vestita & Colibrí de calzones & $\mathrm{RO}, \mathrm{PC}, \mathrm{R}, \mathrm{CT}$ & $\mathrm{B}, \mathrm{M}, \mathrm{F}$ & $\mathrm{S}$ & \\
\hline TROCHILIDAE & Eriocnemis cupreoventris $^{(a)}$ & Colibrí de calzones & $\mathrm{RO}, \mathrm{R}$ & B & $\mathrm{S}$ & \\
\hline TROCHILIDAE & Lafresnaya lafresnayi & Colibrí, Tomineja & $\mathrm{RO}, \mathrm{PC}, \mathrm{R}$ & $\mathrm{B}$ & $\mathrm{S}$ & alt \\
\hline TROCHILIDAE & Pterophanes cyanopterus $^{(a)}$ & Colibrí, Tomineja & $\mathrm{RO}, \mathrm{PC}, \mathrm{R}$ & $\mathrm{B}, \mathrm{F}, \mathrm{VE}$ & $\mathrm{S}$ & \\
\hline PICIDAE & Colaptes rivolii $^{(a)}$ & Carpintero Rojo & $\mathrm{RO}, \mathrm{PC}$ & $\mathrm{B}, \mathrm{M}, \mathrm{F}$ & & \\
\hline GRALLARIDAE & Grallaria squamigera $^{(a)}$ & Tororoi Ondulado & $\mathrm{RO}, \mathrm{PC}, \mathrm{CT}$ & B & AS & \\
\hline GRALLARIDAE & Grallaria ruficapilla $^{(a)}$ & Comprapán & RO & M & $\mathrm{S}$ & \\
\hline GRALLARIDAE & Grallaria rufula $^{(a)}$ & Tororoi rufo & $\mathrm{RO}, \mathrm{PC}, \mathrm{CT}$ & $\mathrm{B}$ & AS & \\
\hline RHINOCRYPTIDAE & Scytalopus latrans ${ }^{(a)}$ & Tapaculo & $\mathrm{PC}$ & B & $\mathrm{S}$ & \\
\hline RHINOCRYPTIDAE & Scytalopus griseicollis ${ }^{(a)}$ & Tapaculo Vientriplateado & $\mathrm{RO}, \mathrm{PC}$ & B & $\mathrm{S}$ & \\
\hline FURNARIIDAE & Margarornis squamiger $^{(a)}$ & Trepadorcito & $\mathrm{RO}, \mathrm{PC}$ & $\mathrm{B}, \mathrm{M}$ & AS & \\
\hline FURNARIIDAE & Hellmayrea gularis $^{(a)}$ & Chamicero & $\mathrm{RO}, \mathrm{PC}, \mathrm{R}$ & $\mathrm{B}$ & AS & \\
\hline TYRANNIDAE & Mecocerculus leucophrys ${ }^{(a)}$ & Atrapamoscas & $\mathrm{RO}, \mathrm{PC}, \mathrm{R}$ & $\mathrm{B}, \mathrm{M}, \mathrm{F}$ & $\mathrm{S}$ & \\
\hline TYRANNIDAE & Ochthoeca fumicolor & Pitajo Ahumado & $\mathrm{RO}, \mathrm{PC}$ & $\mathrm{B}, \mathrm{F}$ & $\mathrm{S}$ & \\
\hline HIRUNDINIDAE & Pygochelidon cyanoleuca $^{(a)}$ & Golondrina & RO & $\mathrm{F}$ & & lat, inr \\
\hline HIRUNDINIDAE & Orochelidon murina ${ }^{(a)}$ & Golondrina & RO & $\mathrm{B}, \mathrm{M}$ & & \\
\hline HIRUNDINIDAE & Riparia riparia & Golondrina & RO & $\mathrm{F}$ & & lat, inr \\
\hline TROGLODYTIDAE & Troglodytes aedon & Cucarachero & $\mathrm{RO}, \mathrm{PC}, \mathrm{R}$ & $\mathrm{B}, \mathrm{M}, \mathrm{F}$ & & \\
\hline TROGLODYTIDAE & Cistothorus platensis $^{(a)}$ & Sotorrey de pajonal & $\mathrm{RO}, \mathrm{PC}$ & $\mathrm{F}$ & $\mathrm{S}$ & \\
\hline TURDIDAE & Turdus fuscater & Siote & $\mathrm{RO}, \mathrm{PC}, \mathrm{CT}$ & $\mathrm{B}, \mathrm{M}, \mathrm{F}, \mathrm{VE}, \mathrm{P}$ & & \\
\hline THRAUPIDAE & Hemispingus superciliaris $^{(a)}$ & Hemispingo superciliado & $\mathrm{RO}, \mathrm{PC}$ & $\mathrm{B}$ & $\mathrm{S}$ & \\
\hline THRAUPIDAE & Anisognathus igniventris & Clarinero & $\mathrm{RO}, \mathrm{PC}, \mathrm{R}$ & $\mathrm{B}, \mathrm{M}, \mathrm{F}, \mathrm{VE}$ & $\mathrm{S}$ & \\
\hline THRAUPIDAE & Tangara vassorii ${ }^{(a)}$ & Azulejo de montaña & $\mathrm{PC}$ & $\mathrm{B}$ & $\mathrm{S}$ & \\
\hline THRAUPIDAE & Conirostrum sitticolor ${ }^{(a)}$ & Picocono dorsiazul & $\mathrm{RO}, \mathrm{PC}$ & $\mathrm{B}, \mathrm{M}$ & $\mathrm{S}$ & \\
\hline THRAUPIDAE & Conirostrum rufum ${ }^{(a)}$ & Picocono rufo & $\mathrm{RO}, \mathrm{PC}$ & $\mathrm{B}, \mathrm{M}$ & $\mathrm{S}$ & \\
\hline THRAUPIDAE & Diglossa lafresnayii & Carbonero brillante & $\mathrm{RO}, \mathrm{PC}, \mathrm{R}$ & $\mathrm{B}$ & $\mathrm{S}$ & \\
\hline THRAUPIDAE & Diglossa humeralis $(a)$ & Carbonero común & $\mathrm{RO}, \mathrm{PC}, \mathrm{R}$ & $\mathrm{B}, \mathrm{M}, \mathrm{F}, \mathrm{VE}$ & & \\
\hline THRAUPIDAE & Diglossa cyanea $^{(a)}$ & Azulejo de montaña & $\mathrm{RO}, \mathrm{PC}$ & $\mathrm{B}, \mathrm{M}, \mathrm{F}, \mathrm{VE}$ & $\mathrm{S}$ & \\
\hline THRAUPIDAE & Catamenia homochroa ${ }^{(a)}$ & Chisga de páramo & $\mathrm{RO}, \mathrm{PC}$ & $\mathrm{F}$ & $\mathrm{S}$ & \\
\hline EMEBERIZIDAE & Arremon torquatus $^{(a)}$ & Gorrión de bosque & $\mathrm{RO}, \mathrm{PC}, \mathrm{CT}$ & $\mathrm{B}, \mathrm{M}$ & $\mathrm{S}$ & \\
\hline EMEBERIZIDAE & Zonotrichia capensis & Copetón & $\mathrm{RO}, \mathrm{PC}$ & $\mathrm{B}, \mathrm{M}, \mathrm{F}, \mathrm{VE}, \mathrm{A}$ & & \\
\hline EMEBERIZIDAE & Atlapetes schistaceus $^{(a)}$ & Gorrión de bosque & $\mathrm{RO}, \mathrm{PC}$ & $\mathrm{B}, \mathrm{M}$ & $\mathrm{S}$ & \\
\hline EMEBERIZIDAE & Atlapetes pallidinucha ${ }^{(a)}$ & Gorrión de bosque & $\mathrm{RO}, \mathrm{PC}$ & $\mathrm{B}, \mathrm{M}$ & $\mathrm{S}$ & \\
\hline PARULIDAE & Setophaga fusca $(a)$ & Reinita gorguinaranja & RO & B & $\mathrm{S}$ & lat, inr \\
\hline PARULIDAE & Myiothlypis nigrocristata $^{(a)}$ & Reinita coroninegra & $\mathrm{RO}, \mathrm{PC}$ & $\mathrm{B}, \mathrm{M}$ & $\mathrm{S}$ & \\
\hline PARULIDAE & Myioborus ornatus $^{(a)}$ & Candelita frentidorada & $\mathrm{RO}, \mathrm{PC}$ & $\mathrm{B}, \mathrm{M}$ & $\mathrm{S}$ & \\
\hline ICTERIDAE & Sturnella magna & Chirlobirlo & $\mathrm{RO}, \mathrm{PC}$ & $\mathrm{P}, \mathrm{A}$ & & \\
\hline FRINGILLIDAE & Sporagra spinescens & Chisga de páramo & RO & $\mathrm{B}, \mathrm{P}$ & & \\
\hline
\end{tabular}


W. Medina et al.

Tabla. Anexo 2.

\begin{tabular}{|c|c|c|c|c|c|}
\hline Orden & Familia & Especie & Nombre común & Método de registro & Cobertura \\
\hline DIDELPHIMORPHIA & Didelphidae & Didelphis pernigra & Fara & CT, T, E & B \\
\hline SORICOMORPHA & Soricidae & Cryptotis thomasi $^{(\mathrm{a})}$ & Musaraña & $\mathrm{TC}, \mathrm{S}$ & $\mathrm{F}, \mathrm{B}$ \\
\hline CINGULATA & Dasypodidae & Dasypus novemcinctus & Armadillo & $\mathrm{R}, \mathrm{E}$ & $\mathrm{CH}, \mathrm{V}$ \\
\hline RODENTIA & Cricetidae & Thomasomys niveipes $^{(\mathrm{a})}$ & Ratón & $\mathrm{S}, \mathrm{TC}, \mathrm{CT}$ & F, B, P, VE \\
\hline RODENTIA & Cricetidae & Microryzomys minutus $^{(a)}$ & Ratón & TC, S & $\mathrm{F}, \mathrm{B}, \mathrm{VE}$ \\
\hline RODENTIA & Cricetidae & Neomicroxus bogotensis & Ratón & $\mathrm{S}$ & $\mathrm{F}$ \\
\hline RODENTIA & Cricetidae & Oligoryzomys fulvescens & Ratón & $\mathrm{S}$ & $\mathrm{F}$ \\
\hline RODENTIA & Caviidae & Cavia aperea & Curí & $\mathrm{CT}, \mathrm{R}, \mathrm{O}, \mathrm{E}$ & $\mathrm{F}, \mathrm{B}, \mathrm{P}, \mathrm{A}, \mathrm{H}, \mathrm{M}$ \\
\hline RODENTIA & Cuniculidae & Cuniculus taczanowskii & Tinajo & CR, E & $\mathrm{B}, \mathrm{M}$ \\
\hline CHIROPTERA & Vespertilionidae & Histiotus montanus & Murciélago & $\mathrm{O}$ & $\mathrm{V}$ \\
\hline LAGOMORPHA & Leporidae & Sylvilagus brasiliensis & Conejo sabanero & $\mathrm{R}, \mathrm{E}$ & $\mathrm{P}, \mathrm{CH}, \mathrm{V}$ \\
\hline CARNIVORA & Canidae & Urocyon cinereoargenteus & Zorro gatuno & E, CT & $\mathrm{V}, \mathrm{B}, \mathrm{VE}$ \\
\hline CARNIVORA & Mustelidae & Mustela frenata & Comadreja & $\mathrm{S}, \mathrm{O}$ & $\mathrm{F}, \mathrm{CH}, \mathrm{A}, \mathrm{M}$ \\
\hline CARNIVORA & Procyonidae & Nasuella olivacea & Guache, runcho & $\mathrm{CT}, \mathrm{R}, \mathrm{O}, \mathrm{A}$ & $\mathrm{B}, \mathrm{F}, \mathrm{A}, \mathrm{CH}, \mathrm{M}$ \\
\hline
\end{tabular}

